the direction of the waves with the velocity of light. These charges lie within a cylinder meeting the plane of $y z$ on the boundary of A. The magnetic vector is perpendicular to the electric vector and equal to it in magnitude both inside and outside the electric charges. The Maxwell-Lorentz equations are satisfied everywhere, and the fact that we are able to specify a type of radiation the total energy of which is proportional to the maximum frequency $p c=\nu$ indicates that quantum theory may be quite compatible with these equations. That all frequencies up to $p c$ occur is seen at once from the equation $\frac{\sin p x}{x}=\int_{0}^{p} \cos q x \cdot d q$. We are justified in regarding this type of field as elementary because, as Levi-Civita pointed out many years ago (Comptes rendus, t. 145, 1907), the electromagnetic force on the moving electricity vanishes everywhere and so the electricity moves freely under no forces and no forces are needed to keep it intact. In this connection it may be mentioned that the force of type $2 \psi \nabla\left(\rho \sqrt{\mathrm{I}-\overline{v^{2}} / c^{2}}\right)$, which has been used to balance the electric force in a suggested model of a stationary electron (Physical Review, September I922), is also zero in the present case because $\psi=0$ and $v^{2}=c^{2}$.

Superposing two quantum fields with

$f=\frac{\sin (p+d p)(x-c t)}{x-c t}$ and $f=-\frac{\sin p(x-c t)}{x-c t}$, respectively,

and with coincident cylinders (or light-darts, to use Silberstein's term), we obtain a wave of nearly homogeneous radiation of total energy $\pi \mathrm{W} d p$. When the light-darts in the two fields are separate entities but close together, the total field still represents an approximately homogeneous type of radiation, but it also possesses some of the properties of a quantum radiation because the light-darts can be regarded as independent and one of them can be captured by an atom and its energy $h v$ absorbed while the other one escapes.

The composite field will behave like radiation of frequencies lying between $\nu$ and $\nu+d \nu$ when reflected and refracted if the elementary quantum-radiation behaves like light of frequency $\nu=c p$.

To test this point we have considered the reflection of our elementary quantum radiation at the surface of the moving mirror $x=u t$. The reflected wave proves to be one of the same type as the first, the electric vector being $-f(x+c t) \mathrm{F}$, where $f(x)=\frac{\sin p^{\prime} x}{x}$ and $p^{\prime}=p \frac{c-u}{c+u}$.

Thus Doppler's principle holds just as if $p c$ were the frequency of homogeneous light instead of the maximum frequency contained in the quantum radiation. The energy relation $\epsilon^{\prime}=h \nu^{\prime}$ still holds and it looks as if W could be regarded as a universal constant $h c / \pi$.

It should be remarked that the elementary radiant field considered here is simply a particular case of a more general type of simple radiant field in which the rays and light-darts issue from a moving point. An ordinary type of electromagnetic field can be built up by superposing two or more simple radiant fields of this type and proceeding to the limit. If, then, ordinary electromagnetic fields are to be regarded as composite, there is nothing strange in regarding an approximately homogeneous type of radiation as composite.

H. BATEMAN

California Institute of Technology, Pasadena, California, March 5 .

\section{Spermatogenesis of the Lepidoptera.}

I sHould be glad of the hospitality of the columns of NATURE to reply to two observers whose papers in the December number of the Quarterly Jounnal of Microscopical Science have only recently come under my attention. Dr. Robert H. Bowen, of Columbia University, has investigated the spermatogenesis of the Lepidoptera, a subject which formed the first part of my series of papers on the "Cytoplasmic Inclusions of the Germ Cells." His account differs from previous ones chiefly in two respects-he states that the mitochondrial part of the spermatid is not a skein or spireme but a plate-work, and what is a much more interesting objection, he denies the previous descriptions of the metamorphosis of this skein into a tail-sheath, and instead describes it as degenerating, and the tail region being formed of a new central substance.

In the same Journal, Mr. Graham Cannon has redescribed the louse mitosome, and supports Dr. Bowen's conclusion that this body is not a skein but a plate-work. Dr. Bowen agrees with me so far as the general appearance of the material is concerned but in a long discussion brings up a number of reasons for supposing that the body in question is a platework something roughly like the head of a fancy chrysanthemum. Mr. Graham Cannon has also given a similar and short account of his reasons for supposing that this body is actually a plate-work formed by a system of vacuoles.

Some years ago when Prof. Doncaster was writing his latest book, he came to see the material illustrating my view that the acrosome is always formed in association with the Golgi apparatus. He was shown my preparations of Smerinthus testes, and objected then to my description of the mitosome or nebenkern as a spireme. Dr. Bowen and Mr. Cannon will be glad to know this. However, I never found any reason to alter my views, even with such distinguished opposition, because it seemed to me that whether the "spireme" was formed of a flat ribbon, or a round, string, it was actually pulled out as the spermatid lengthened, much like a ball of string. The figure formed by the mitochondria of the spermatid is not a matter of importance so far as concerns the larger questions surrounding the study of the cytoplasmic inclusions.

When, however, we turn to the second objection brought forward by Dr. Bowen, we find a matter of considerable importance. Dr. Bowen's " central substance" was believed to be the partly unravelled or pulled-out mitochondrial skein; it is figured by me in Plate 25, fig. 47, of my paper. His account of this new substance being something apart from the nebenkern or mitosome, and of the latter not taking direct part in the formation of the tail, is worthy of reinvestigation.

Except for Dr. Bowen's new interpretation of the formation of the sperm tail, he adds nothing new to our knowledge of the spermatogenesis of the Lepidoptera. His account is valuable, however, because of the fact that it confirms my drawings of the appearances of the material already described by me. Some of his spermatid cells are effete and drawn from bundles which are in the process of formation of atypic sperms. The whole question will be dealt with by me in a full account elsewhere. I merely take this early opportunity of stating my position.

Mr. Graham Cannon's statements will also be examined at length elsewhere.

Zoology Department,

Dublin University, April 9.

NO. 279 I, VOL. I I I ] 